A N N A L E S Annales de Bretagne et des Pays de l'Ouest

\title{
Les poches de l'Atlantique 1944-1945. Le dernier acte de la Seconde Guerre mondiale en France
}

Fabien Lostec

\section{(2) OpenEdition}

Édition électronique

URL : https://journals.openedition.org/abpo/6632

DOI : $10.4000 / a b p o .6632$

ISSN : 2108-6443

\section{Éditeur}

Presses universitaires de Rennes

\section{Édition imprimée}

Date de publication : 10 décembre 2020

Pagination : 231-234

ISBN : 978-2-7535-8225-5

ISSN : 0399-0826

\section{Référence électronique}

Fabien Lostec, « Les poches de l'Atlantique 1944-1945. Le dernier acte de la Seconde Guerre mondiale en France ", Annales de Bretagne et des Pays de l'Ouest [En ligne], 127-4 | 2020, mis en ligne le 10 décembre 2020, consulté le 06 janvier 2023. URL : http://journals.openedition.org/abpo/6632 ; DOI : https://doi.org/10.4000/abpo.6632 
fut un correspondant assidu des pupilles pendant la Guerre.

On apprend aussi que parmi les 165 pupilles morts pour la France, 84 soit la moitié ont été des enfants abandonnés, 51 des moralement abandonnés (30,1\%) et 26 des orphelins (15,8\%), alors même que les nouveaux admis en 1900 sont pour les trois quarts des enfants abandonnés et que les moralement abandonnés ne représentent que 13,5\% des pupilles et les orphelins 10,5\%. L'âge d'entrée à l'Assistance Publique apporte aussi de précieux renseignements. Seuls $4 \%$ des soldats ont été accueillis avant un mois et $12 \%$ entre un mois et un an. On sait pourtant par ailleurs que parmi les enfants abandonnés, de loin les plus nombreux des pupilles, huit sur dix arrivent avant un mois. Ce décalage avec les données relatives aux soldats décédés soulignent combien était importante la mortalité infantile des enfants trouvés et abandonnés, pupilles arrivés très tôt à l'Assistance Publique. Il est rappelé qu'après leurs premières années en nourrice, les pupilles de l'époque sont gagés dès treize ans, généralement comme pâtres puis domestiques agricoles. 134 des 165 pupilles exercent d'ailleurs un métier agricole. Il est aussi souligné combien l'Assistance Publique se veut gardienne du secret : les origines ne sont pas révélées et il n'est pas permis de correspondance avec la famille, même pendant la guerre.

Les pupilles et anciens pupilles ont payé un lourd tribut à la Nation. Le plus jeune est mort à dix-huit ans, le plus âgé à quarante-huit. La très grande majorité des soldats honorés ont fait la guerre comme hommes de troupe. Presque tous appartiennent à l'infanterie, arme la plus décimée dans le conflit. En 1919, l'inspecteur recensait sur 380 pupilles et anciens pupilles mobilisés, 65 morts au champ d'honneur, soit $21 \%$ de tués, 110 blessés, 15 disparus et 22 faits prisonniers.

La reproduction de documents enrichit agréablement les textes, tout au long de l'ouvrage et dans l'annexe "illustration ». Il s'agit en particulier de lettres et de cartes postales adressées à l'inspecteur. L'Assistance avait organisé un service spécial pour la correspondance avec les pupilles militaires. Certains courriers sont assez convenus et portent sur la demande d'un pécule ou le remerciement de son octroi. D'autres se rapprochent davantage d'une lettre personnelle adressée à un proche. Certains adoptent un ton très guerrier : " Je me suis engagé pour tuer et éventrer la chair non civilisée ", écrit l'un; "Je vous assure que les boches reçoivent quelque chose comme pruneau ", témoigne un autre. D'autres courriers évoquent davantage les sentiments éprouvés : "La guerre est longue surtout pour nous qui n'avons ni parents ni aucune ressource ". D'autres encore, ou les mêmes, font part des conditions matérielles, comme par exemple le manque de cidre. Des photos nous rendent quelques-uns encore plus présents. Certaines comme celle d'Édouard Bandily ont été prises alors qu'ils étaient enfants. Mais les autres nous les montrent en soldats, à l'exemple de celle de Louis Dévédec en uniforme de zouave ou d'Eugène Bunel qui pose en août 1917. Yves Raimbault, quant à lui, arbore sa croix de guerre.

Cet ouvrage plein d'enseignements et très vivant est un très bel hommage à ces jeunes hommes et à leur courage.

Martine FAUCONNIER CHABALIER

Catala, Michel (dir.), Les poches de l'Atlantique 1944-1945. Le dernier acte de la Seconde Guerre mondiale en France, Rennes, Presses universitaires de Rennes, coll. « Histoire », 2019, 324 p.

Le " front des oubliés ", c'est ainsi que dans le sud de la Bretagne l'on nomme les combats ayant eu lieu pour libérer les poches de l'Atlantique. L'ouvrage qui lui 
est ici consacré rassemble les actes d'un colloque organisé par Michel Catala en mai 2015, à l'occasion de la commémoration du $70^{\mathrm{e}}$ anniversaire de la libération de la poche de Saint-Nazaire. Composé d'une vingtaine d'articles et d'un livret photographique de six pages, il éclaire d'un jour nouveau une histoire longtemps restée dans l'ombre, complétant intelligemment le travail de Stéphane Simonnet (Les poches de l'Atlantique. Les batailles oubliées de la Libération, janvier 1944-mai 1945, Paris, Tallandier, 2019 [2015]). L'objectif est affiché d'emblée : proposer une histoire globale des poches, de leur constitution à leur mémoire, en passant par leur relèvement, sans négliger la diversité des situations locales (p. 8). Pour ce faire, l'ensemble est divisé en cinq parties, introduites par Hervé Drévillon qui, en s'intéressant au temps long de la guerre de siège, met en évidence le principe d'intégrité territoriale comme préalable à la compréhension des enjeux de la réduction de ces môles de résistance allemande (p. 13).

Pour débuter, les auteurs présentent le contexte de formation ainsi que l'histoire des poches. Les enjeux et le déroulement de la libération de la Bretagne permettent notamment de mieux comprendre la constitution de celles de Lorient et de SaintNazaire, en août 1944. Bon nombre de résistants s'engagent alors dans la nouvelle armée française et poursuivent la guerre jusqu'en mai 1945 (Christian Bougeard). Dès lors que les forces alliées, qui foncent vers l'Allemagne, n'en font pas une priorité, le front de l'Atlantique est effectivement tenu par des troupes essentiellement formées de combattants français. Après avoir projeté leur libération prochaine, les civils présents dans les poches se sentent quant à eux abandonnés. Du fait des pénuries, ils deviennent de plus en plus " encombrants " aux yeux de l'occupant (Jacqueline Sainclivier). Dans le Sud-Ouest, le vécu des différents protagonistes est très fragmenté, que ce soit entre ou à l'intérieur des forteresses (Festungen) de La Rochelle et de la Gironde. Malgré les discussions entre les Allemands et les Forces françaises de l'Ouest, les poches sont marquées par les violences de la guerre totale, dont la principale manifestation reste le bombardement de Royan en janvier 1945 (Éric Kocher-Marboeuf). Dans tous les cas, les historiens remarquent la perméabilité du front, du fait des percées allemandes pour se ravitailler et des évacuations de civils.

Une deuxième partie est consacrée aux enjeux que représentent les poches, qu'ils soient politiques, militaires ou économiques. Après avoir vu leurs échanges être réduits à néant durant l'Occupation, celles-ci ne peuvent relancer leur activité commerciale en 1944. Mais ce problème ne leur est pas spécifique, comme le démontre une comparaison avec les ports non assiégés. Détruits par l'occupant, tous doivent attendre la reconstruction de leur potentiel technique et économique pour voir leur trafic repartir (Bruno Marnot). De la même manière, il est important de relativiser l'importance stratégique du front de l'Atlantique, comme le démontre l'étude des bases de sous-marins de Lorient, Saint-Nazaire et La Pallice. Modernes et fonctionnelles, elles jouent certes un rôle primordial de 1941 à 1943 et après 1945, mais durant l'empochement, leur utilité stratégique pour la marine allemande s'avère négligeable (Jean-Baptiste Blain). Les poches étant très bien défendues au sol, c'est bien l'intervention des forces aériennes qui est déterminante. Aidées par la puissance de feu des Alliés, les Forces aériennes de l'Atlantique sont alors commandées par le général Corniglion-Molinier. Malgré d'inévitables tensions, celui-ci parvient à unir des troupes hétérogènes et, ainsi, à préfigurer l'amalgame de la nouvelle armée de l'air. Surtout, il démontre que les forces françaises sont capables de mener des opérations aériennes d'importance (Jérôme de Lespinois). De fait, les poches sont principalement un enjeu politique, notamment pour le général de Gaulle, qui a besoin d'une victoire pour s'imposer face aux Alliés. Dans le même temps, les Français sont dépendants des forces américaines et la situation des poches ne peut évoluer que si, à l'Est, il y a quelque chose de nouveau (Christine Levisse-Touzé). 
Une troisième partie examine la vie quotidienne des assiégés et des assiégeants. En dépit d'une grande diversité de situations, les empochés possèdent une identité commune qui, outre l'isolement et la difficulté de vivre dans les poches, est construite sur un sentiment d'injustice. Les empochés sont en effet doublement suspects. D'un côté, les Allemands font usage d'une grande violence à l'égard de ceux qui résistent et, plus largement, à l'égard des civils vus comme des " terroristes " en puissance. De l'autre, la population française les accuse de collaborer avec l'occupant (Daniel Sicard et Jean-Claude Catherine). Pour les libérer, les combattants français viennent de partout : de l'étranger, d'outre-mer et, surtout, de métropole. Dans un premier temps, la dynamique régionale de leur recrutement est cependant manifeste. Mais en raison des pertes d'effectifs qui s'expliquent moins par le combat stricto sensu que par les maladies, les accidents ou la réorganisation des forces armées, la géographie du recrutement a ensuite tendance à s'élargir (Stéphane Weiss). Durant cette période de fusion avec l'armée régulière, les FFI peuvent être en mal de reconnaissance. C'est ce qui apparaît dans la presse résistante charentaise, qui ne cesse pourtant de mettre en avant le sens de l'engagement de ces troupes imprégnées de l'esprit maquisard (Hugues Marquis). En face, l'occupant est accompagné par des troupes venant de l'Est, dont il craint la désertion. Essentiellement composées d'hommes usés et démoralisés, elles apparaissent donc moins comme une ressource que comme une préoccupation pour les Allemands, qui craignent leur défection. S'il ne s'agit pas ici de nier les exactions que ces dernières ont commises sur les civils et les résistants, force est néanmoins de constater que la brutalisation observée à l'Est ne revêt pas la même intensité à l'Ouest (Frédéric Dessberg).

L'ouvrage s'intéresse ensuite à la libération et au relèvement des poches. Dans cette perspective, le mémento de l'état-major du détachement de l'armée de l'Atlantique se révèle primordial. Rédigé dans le contexte incertain de la poursuite de la guerre, il révèle le travail de planification qu'effectue l'armée française. À la fois militaire et politique, ce document prévoit la mise en place de l'état de siège pour réinstaller l'autorité républicaine. Tout est fait pour éviter les débordements de l'été 1944 et sécuriser les territoires ainsi que les individus libérés, qu'ils soient allemands ou français (Michaël Bourlet). Ce plan de réoccupation des poches n'oublie pas le châtiment des collaborateurs. L'ombre portée de la Libération explique là encore les spécificités de l'épuration menée à la suite de l'effondrement de la poche de SaintNazaire. La répression ayant pu être anticipée, elle est plus encadrée qu'en 1944. Un encadrement d'autant plus nécessaire que la libération de la poche coïncide avec la seconde vague d'épuration extrajudiciaire alors observée en France, elle-même liée au rapatriement des prisonniers de guerre, des déportés et des requis. Mais si le contraste entre répression projetée et répression réalisée est moins prononcé que quelques mois auparavant, l'épuration doit être accélérée en raison des délais de saisie des cours de justice. L'épuration apparait finalement relativement modérée, sauf à l'encontre des profiteurs de guerre et des femmes (Marc Bergère). Aidé par la bonne entente entre le préfet et le Comité départemental de Libération, l'État parvient à mettre en place une structure administrative provisoire, ce qui lui permet d'organiser simultanément les élections municipales et cantonales. Mais à l'image de ce qui se passe dans le reste de la Loire-Inférieure, ces scrutins ne bouleversent pas la physionomie politique d'avant-guerre : les campagnes demeurent conservatrices et Saint-Nazaire vote à gauche (Jean-François Stéphan). Dans le même temps s'amorce la reconstruction des villes de l'Atlantique. Après avoir longtemps été dépeintes comme des espaces reconstruits sans originalité, leur image est aujourd'hui valorisée. Progressivement, elles sont reconnues pour avoir été les laboratoires des théories urbanistiques du xxe siècle (Daniel Le Couédic). 
Pour finir, une cinquième partie fait un sort à la question de la mémoire. Notons tout d'abord que les poches ont très peu intéressé la littérature. L'ouvrage d'Henri Rio-Le Mesle, paru en 1949, Les oubliés du Tréhic, est une exception. En réalité, l'auteur profite d'un arrière-plan romanesque pour dénoncer la collaboration de certains habitants du Croisic. Son "épuration de papier " est censée corriger une épuration judiciaire qu'il juge trop clémente. Réquisitoire contre les notables, cette publication est également un outrage à la population qui, en étant décrite comme s'étant accommodée de l'empochement, se voit déniée son statut de victime (JeanBaptiste Bruneau). En Bretagne sud, les lieux de mémoire sont essentiellement les blockhaus. Mais ils ont de plus en plus tendance à disparaître du fait de l'urbanisation et de l'accélération de l'activité touristique. Même si leur intérêt historique et mémoriel est progressivement reconnu, leur patrimonialisation est difficile car les blockhaus demeurent perçus comme des kystes défigurant le littoral, y compris par les institutions publiques (Christophe Cérino). Les lieux de mémoire prennent enfin la forme de monuments commémoratifs. Épousant peu ou prou le syndrome de Vichy analysé par Henry Rousso, la mémoire collective des poches est marquée par trois phases. Après les célébrations solennelles de l'immédiat après-guerre, les commémorations s'essoufflent dans les années 1960-1970, avant de connaître un net regain d'intérêt à compter des années 1980. À l'exception notable de la ville de Royan, toujours marquée par le bombardement de janvier 1945, les villes organisent des commémorations de plus en plus festives, où l'identité locale prend le pas sur la dimension nationale. Parallèlement une double dynamique d'héroïsation des victimes et de victimisation des héros participe à la construction d'une communauté du passé, gage d'une communauté de destin (Rémi Fabre et Yves Jaouen).

$\mathrm{Au}$ bout du compte, la force du livre réside dans sa capacité à varier les approches et les échelles d'analyse. Quelques redites existent certes d'une communication à l'autre, mais rien qui perturbe la lecture. Les textes se répondent et on ne peut qu'apprécier un ensemble qui fait sens et ouvre de nouvelles pistes de réflexion. Et ne nous y trompons pas : si le sous-titre indique que les poches de l'Atlantique forment "le dernier acte de la Seconde Guerre mondiale ", elles ne sont pas pour autant analysées comme son épilogue. En cela, l'ouvrage participe à l'actuel renouvellement historiographique des sorties de guerre, démontrant parfaitement que le mouvement de pacification n'est ni inéluctable, ni linéaire, mais, au contraire, rythmé par des "secousses " plus ou moins violentes et complexes.

Fabien LosTEC 